\title{
On the Quasi-stationary curving dynamics of a railroad truck
}

True, Hans; Hansen, T. G.; Lundell, H.

Published in:

Proceedings of the 2005 ASME/IEEE Joint Rail Conference, Pueblo, Colorado

Publication date:

2005

Document Version

Publisher's PDF, also known as Version of record

Link back to DTU Orbit

Citation (APA):

True, H., Hansen, T. G., \& Lundell, H. (2005). On the Quasi-stationary curving dynamics of a railroad truck. In Proceedings of the 2005 ASME/IEEE Joint Rail Conference, Pueblo, Colorado (pp. 131-138). IEEE.

\section{General rights}

Copyright and moral rights for the publications made accessible in the public portal are retained by the authors and/or other copyright owners and it is a condition of accessing publications that users recognise and abide by the legal requirements associated with these rights.

- Users may download and print one copy of any publication from the public portal for the purpose of private study or research.

- You may not further distribute the material or use it for any profit-making activity or commercial gain

- You may freely distribute the URL identifying the publication in the public portal

If you believe that this document breaches copyright please contact us providing details, and we will remove access to the work immediately and investigate your claim. 


\section{ON THE QUASI-STATIONARY CURVING DYNAMICS OF A RAILROAD TRUCK}

\author{
Hans True \\ Informatics and Mathematical Modelling \\ The Technical University of Denmark \\ DK-2800 Kgs.Lyngby \\ Denmark \\ Email: ht@imm.dtu.dk
}

\author{
T.G.Hansen \\ IMM \\ The Technical University of Denmark \\ DK-2800 Kgs.Lyngby \\ Denmark
}

\author{
H.Lundell \\ IMM \\ The Technical University of Denmark \\ DK-2800 Kgs,Lyngby \\ Denmark
}

\begin{abstract}
We examine three aspects of the dynamics of the Cooperrider truck travelling in a curve with constant radius. First the critical speed is found. Second we investigate the existence of multiple steady solutions to the curving problem. Third - and it is related to the second problem - we shall examine the position of the truck frame and the wheelsets during curving. One interesting result is that for a given superelevation there exist curve radii for which the critical speed is exceeded, when the vehicle negotiates the curve with the allowed maximum cant deficiency. These critical speeds are lower than the critical speed on straight rack.
\end{abstract}

\section{INTRODUCTION}

All railroad vehicles oscillate borizontally perpendicularly to the track center line, the so-called 'hunting'. when the speed of the vehicle exceeds a certain 'critical speed". The phenomenon has for more than a century erroneously been treated as a tinear stability problem. The dynamics of a travelling railroad vehicle is highly nonlinear, primarily - but not only - due to the wheel/rail contact surface geometry and the nonlinear stress-strain velocity relation in that surface. A survey of the contact problem has been given by Kalker [1].

Many authors have treated the hunting problem. A purely kinematic investigation was presented by Klingel [2], and in the 20 th century several people investigated the dynamic problem. The first person, who correctly formulated the problem of finding the critical speed as a bifurcation problem in nonlinear dynamics, was Huilgol [3]. A survey of linear stability as well as of nonlinear bifurcation analyses was given by Knothe and Böhm [4].

On a straight railroad line the dynamic problem, formulated in a reference frame that moves with the vehicle along the track center line, has a stationary solution. For sufficiently low speed it is unique and asymptotically stable. In the great majority of cases this solution loses stability, when the speed grows, in a subcritical Hopf bifurcation. In the Hopf bifurcation point at the speed $V_{H}$ a new and periodic motion is created - it 'branches off' the already known stationary solution. When the bifurcation is 'subcritical', the new periodic solution exists for speeds lower than $V_{H}$, and it is unstable. The unstable periodic solution gains asymptotic stability at a lower speed in a saddle-node bifurcation, which defines the critical speed $V_{c}$ of the vehicle, because it is the speed at which the stationary solution loses its uniqueness. In the saddle-node bifurcation point the periodic solution turns over towards higher speeds. In the speed interval $\left(V_{c}, V_{H}\right)$ three solutions to our nonlinear dynamical problem exist. Two of them - one stationary and one periodic solution - are asymptotically stable. The initial conditions or the disturbances determine, which of the stable solutions will emerge, therefore the stationary solution is sensitive to finite disturbances above the critical speed $V_{c}$. It means that the stationary motion will jump to the periodic motion, if a disturbance pushes the trajectory of the motion into the domain of attraction of the periodic motion. The dynamics is usually depicted in a bifurcation diagram, which plots the am:plitude of the various solutions versus the control parameter - in 


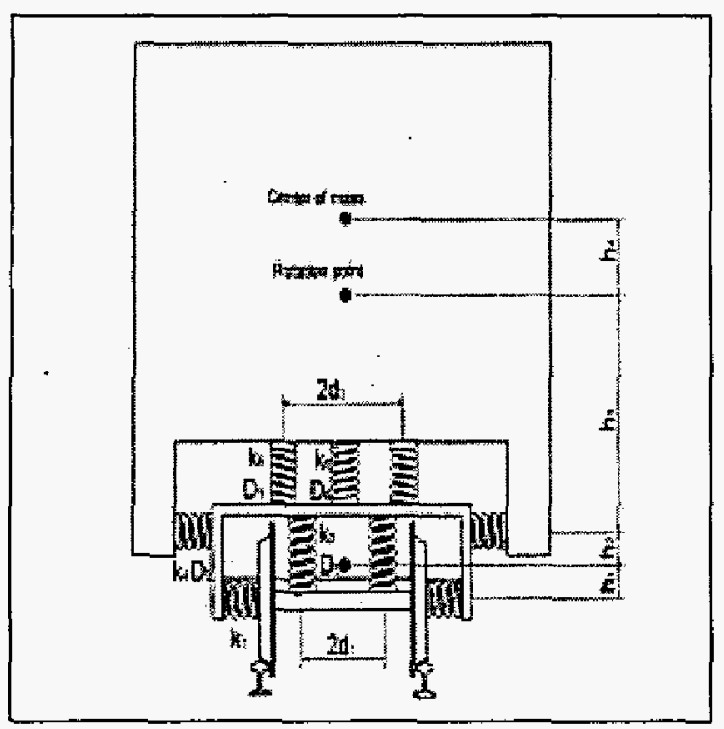

Figure 1. THE COOPERRIDER TRUCK. THE SPRING SIGNATURES INDICATE PURE SPRINGS IF THEY ONLY ARE DENOTED BY A SPAING CONSTANT $k_{i}$, AND A SPRING-DAMPER COMBINATION IF THEY IN ADDITION ARE DENOTED BY A DAMPING CONSTANT $D_{i}$. THE SPRING-DAMPER COMBINATION DENOTED BY $k_{6}$ AND $D_{6}$ ACTS AGAINST THE YAW MOTION OF THE TRUCK FRAME

our problem the speed, $\mathrm{V}$,

On curved track the bifurcation diagram is similar, but True and Birkedal Nielsen [5] found that the critical speed may be lower than on straight track. Petersen and Hoffmann [6] extended their bifurcation analysis to larger curve radii. They found in a student project that a jump of the Hopf bifurcation down to the saddle-node happens for a fixed superelevation and growing curve radius. Then the Hopf bifurcation becomes supercritical. In a supercritical Hopf bifurcation the periodic solution only exists for larger speeds than $V_{H}$, and the new periodic solution is asymptotically stable.

This contribution intends to deepen the understanding of these phenomena and find their dependence on the parameters of the railroad line.

\section{THE VEHICLE MODEL}

We investigate the dynamics of a railroad truck for a passenger car. All the important nonlinearites that influence the critical speed - the suspension elements, the wheel/rail contact geometry and the contact forces - are concentrated in the trucks. We have chosen the so-called 'Cooperrider truck' [7] Fig. 1 and Fig. 2 as our model, because it has been used often as a benchmark model. We have added a yaw damper and pitch dampers to the truck and

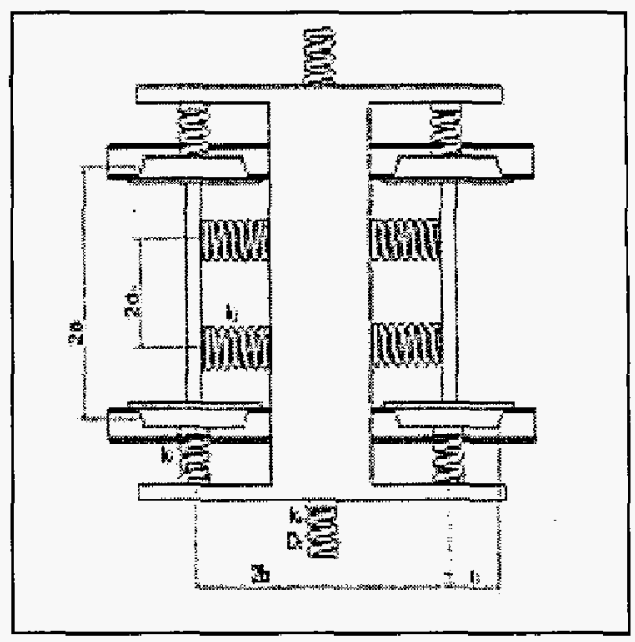

Figure 2. THE COOPERRIDER TRUCK. $k_{i}$ AND $D_{i}$ DENOTE SPRING AND DAMPER CHARACTERISTICS

use wheelsets with the S 1002 wheel profile running on UIC 60 tails, which are laid with a slope of $1 / 40$ towards the track center line. All elements of the truck are assumed to be rigid with the exception of the springs and dampers that have linear characteristics. W. Kik's routine RSGEO is used to calculate the ideal contact points between the wheels and the rails in dependence on the lateral displacement of the wheelset in the track. For the calculation of the normal forces in the wheel-rail contact zone we use Hertz's theory, and the tangential forees are calculated using Shen-Hedrick-Elkins' formula [8]. The truck model has fourteen degrees of freedom and an equation for each wheelset that expresses the condition that the speed of rotation of the wheelset corresponds to the speed of travel of the truck. Due to the number of degrees of freedom and the nonlinearities the dynamic problem is investigated numerically. We use the routine SDJRK as the solver and MATLAB for post processing. SDIRK is an acronym for Singly Diagonally Implicit Runge-Kutta. and it is an efficient solver for stiff problems as ours. We use an error tolerance $10^{-8}$ and an output stepsize of $10^{-3}$. Each branch of the solution is found by a path following procedure. The existence of the Hopf bifurcations is verified by a numerical investigation of the variation of the eigenvalues of the Jacobian in a neighborhood of the bifurcation points.

Before we go over to the next section 'Some results', we shall refresh some important concepts from nonlinear dynamics. For a deeper insight the interested reader is referred to the article by True [9].

Nonlinear dynamic problems often have several steady solutions, where each of them depends on the initial conditions used. This is a contrast to the linear problems that have unique steady 
solutions. A steady solution is defined as the asymptotic limit of the solution for time, $t$, going to infinity - in other words after the transient has died out. A steady solution may be stationary, periodic or have a more complicated structure like chaos. The set of all initial conditions, from which the trajectories approach a steady solution, is called the basin of attraction of that solution. Vehicle dynamic problems are parameter dependent. Of all the parameters the speed. $V$, is particularly interesting, because it varies during the ride, and $\mathrm{V}$ is therefore chosen as the external control parameter in our problem.

The multiplicity of solutions of a nonlinear problem depends on the parameters. In order to obtain an overview we most of ten use bifurcation diagrams, where a quantity characterizing a certain solution is plotted versus the parameter. A typical example from vehicle dynamics is shown in Fig. 7. A broken line denotes an unstable solution. The figure shows a subcritical bifurcation of a periodic solution from a stationary solution, $x=0$, at $V=V_{\text {lincrit }}$ and a saddle-node bifurcation at $V=V_{\text {monlincrit }}$, where the periodic solution turns around and gains asymptotic stability. At a subcritical bifurcation the bifurcating periodic solution coexists with the stable stationary solution, and the periodic solution is unstable. At a supercritical bifurcation the periodic solution coexists with the unstable stationary solution, and it will be stable. Since $V_{\text {nonlincrit }}$ is the speed, at which the stationary solution $V=0$ loses its uniqueness, the stationary solution becomes sensitive to finite disturbances above that speed. The speed $V_{\text {nonlincris }}$ is therefore the critical speed of the vehicle. The stationary solution will remain asymptotically stable, until the vehicle reaches the speed $V_{\text {incrit }}$. Above $V_{\text {lincrit }}$ the stationary solution is unstable, and the only stable solution to the problem - at least in some higher speed interval - is the periodic solution.

Bifurcation points like $V_{\text {lincrit }}$ and $V_{\text {sonlincrit }}$ depend of course on the other parameters in the problem. In curving dynamics we have two important additional parameters, namely the radius of the curve and the superelevation of the track. A plot of the bifurcation points versus one or more of the parameters is called a bifurcation set. An example of a bifurcation set is Fig. 3. It is important to keep the difference between a bifurcation diagram and a bifurcation set in mind, since they may look alike. The bifurcation points in a bifurcation set may merge or tum around or cross - just like the curves in a bifurcation diagram. The bifurcation set, however, illustrates the dependence of the bifurcation points on the parameters. The bifurcation diagram illustrates the dependence of a norm of the solutions eg. the amplitude on the parameters.

\section{SOME RESULTS}

On a straight track the first Hopf bifurcation point for the Cooperrider truck is $117.3 \mathrm{~m} / \mathrm{s}$ and the critical speed is 53.97 $\mathrm{m} / \mathrm{s} \sim 194 \mathrm{~km} / \mathrm{h}$. [6]. These values are not unusual for a modern truck designed for passenger cars with a maximum service speed

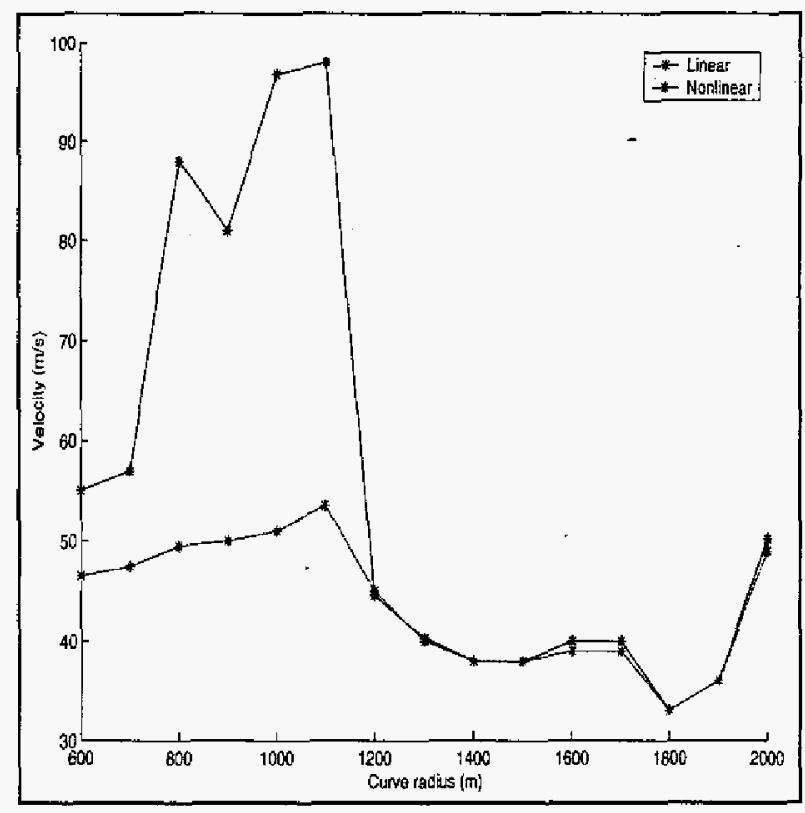

Figure 3. THE CRITICAL SPEEDS FOUND BY PETERSEN AND HOFFMANN [6]. THE SUPERELEVATION IS $2^{\circ}$. NEAR $R=1200 \mathrm{~m} A$ SUBCRITICAL HOPF BIFURCATION SUDDENLY CHANGES TO A SUPERCRITICAL HOPF BIFUACATION

of $160 \mathrm{~km} / \mathrm{h}$. The Hopf bifurcation - in the following called 'the linear critical speed' - and the critical speed - or correspondingly 'nonlinear critical speed' - in curves with a radius in the interval $600 \mathrm{~m}<\mathrm{R}<2000 \mathrm{~m}$ were found by Petersen and Hoffmann [6]. In Fig. 3 we show their results as a bifurcation set for a track with a superelevation of $2^{\circ}$.

We see that the linear critical speed grows - but not monotonously - from $\mathrm{R}=600 \mathrm{~m}$ to $\mathrm{R} \sim 1200 \mathrm{~m}$. The Hopf bifurcation is very sensitive to the wheel-rail contact geometry, which depends on the yaw angle and the lateral displacements of the wheelsets. They in turn depend primarily on the superelevation and the radius in the curve. The nonlinear critical speed also grows, but much slower and monotonously, up until a radius of $R \sim 1100 \mathrm{~m}$. At $R \sim 1100 \mathrm{~m}$ the bifurcation points are located close to their corresponding values on straight track, but then the nonlinear critical speed decreases for growing $R$. Around $\mathrm{R}=1200 \mathrm{~m}$ the linear critical speed jumps down to the nonlinear critical speed, whereby the two bifurcation points merge and create a single supercritical Hopf bifurcation. The critical speed then drops to around $38 \mathrm{~m} / \mathrm{s}$ at $\mathrm{R}=1400 \mathrm{~m}$, but from $\mathrm{R}=1500 \mathrm{~m}$ the linear critical speed and the nonlinear critical speed separate and merge again at $\mathrm{R} \sim 1800 \mathrm{~m}$, where a minimum exists: When $\mathrm{R}$ grows beyond $1800 \mathrm{~m}$, the situation seems to approach the situa-

Copyright (C) 2005 by ASME 


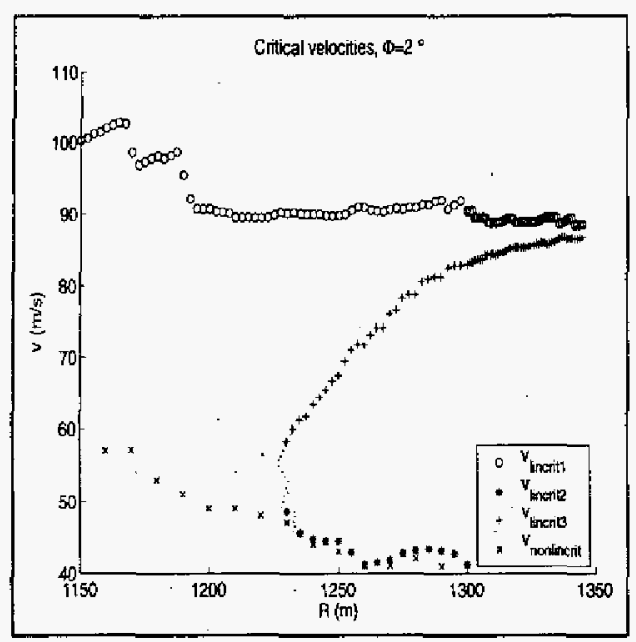

Figure 4. THE BRANCHES OF THE LINEAR (HOPF BIFURCATIONS) AND NONLINEAR CRITICAL SPEEDS

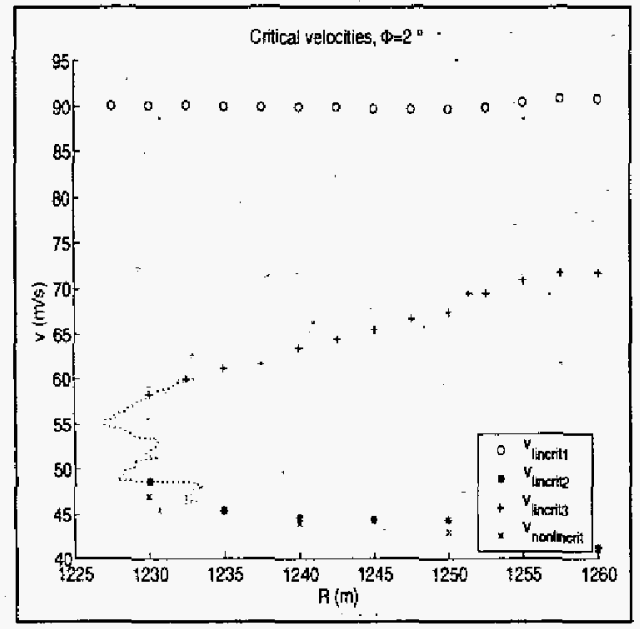

Figure 5. AN ENLARGEMENT OF A SECTION OF FIG.4

tion on straight track, but further analysis is necessary to confirm that postulate.

The nonlinear critical speeds in the diagram are quite large, and it is therefore interesting to test the relevance for a real situation. UIC has set standards for the maximum allowed 'uncompensated lateral acceleration' on railroad lines. From these it is easy to calculate the maximum speed in a curve with a given radius and superelevation. In our example most of the critical speeds will lie above the maximum speed allowed on the basis

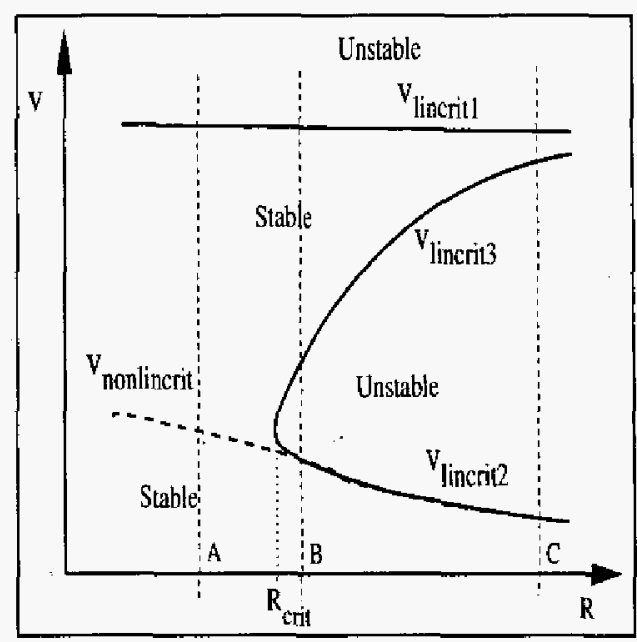

Figure 6. A PLOT SHOWING THE ASYMPTOTICALLY STABLE REGIONS OF THE STATIONARY SOLUTION IN DEPENDENCE ON THE CURVE RADIUS R AROUND R $=1230 \mathrm{~m}$.

of the UIC standard, but it will not be the case for $R=1800 \mathrm{~m}$. If the vehicle travels through that curve with the allowed uncompensated lateral acceleration of $0.67 \mathrm{~m} / \mathrm{s}^{2}$, then it will start to hunt. The critical speed in that curve is only $33 \mathrm{~m} / \mathrm{s} \sim 119 \mathrm{~km} / \mathrm{h}$, whereas the critical speed on straight track was $194 \mathrm{~km} / \mathrm{h}$.

We want to investigate the merger of the nonlinear and linear critical speeds around $R=1200 \mathrm{~m}$. The numerical calculations revealed the bifurcation sets shown in Fig. 4 and Fig. 5. It is seen in Fig. 5 that a merger of two Hopf bifurcation points appears at a speed $\sim 55 \mathrm{~m} / \mathrm{s}$ for $\mathrm{R}=1226.6 \mathrm{~m}$, whereby the lowest linear critical speed jumps down from $90 \mathrm{~m} / \mathrm{s}$. The values are not exactly the same as those in Fig. 3, because the wheel profile has been slightly modified in the model we consider in this work. and there are no pitch dampers in Petersen and Hoffmann's model. The lower branch from the merger, $v_{\text {lincrit } 2}$, wiggles violently around $\mathrm{R}=1230 \mathrm{~m}$, until it merges with $v_{\text {nonincrit }}$ around $\mathrm{R}=1232.4 \mathrm{~m}$ and the speed $V=46.2 \mathrm{~m} / \mathrm{s}$. The diagram in Fig. 6 shows qualitatively the regions of asymptotical stability of the stationary solution in dependence on the curve radius $R$.

In order to depict the merger qualitatively we consider the bifurcation diagrams in the three sections denoted ' $A$ ', 'B' and ' $C$ ' in Fig. 6. In this way the character of the merger will become clear. In Fig. 7 the section ' $A$ ' is shown. The bifurcation diagram is that of a normal subcritical Hopf bifurcation, where the unstable, periodic solution gains stability in a saddle-node bifurcation. In Fig. 8 (section B) we see that two new Hopf bifurcation points $v_{\text {lincrit } 2}$ and $v_{\text {lincrit } 3}$ have appeared in the diagram. They lie below the old $v_{\text {lincrit }}$, which from now on will be denoted $v_{\text {linerit } 1 \text {. }}$ Between $v_{\text {/incrit } 2}$ and $v_{\text {lincrit } 3}$ the stationary solution has become 


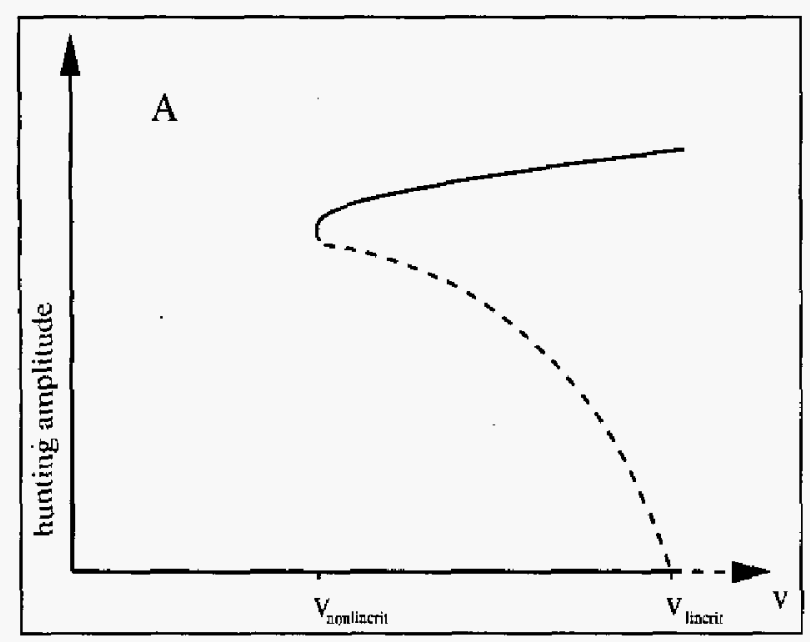

Figure 7. SECTION A FROM FIG.6 SHOWING THE SUBCRITICAL HOPF AND THE SADDLE-NODE BIFURCATIONS

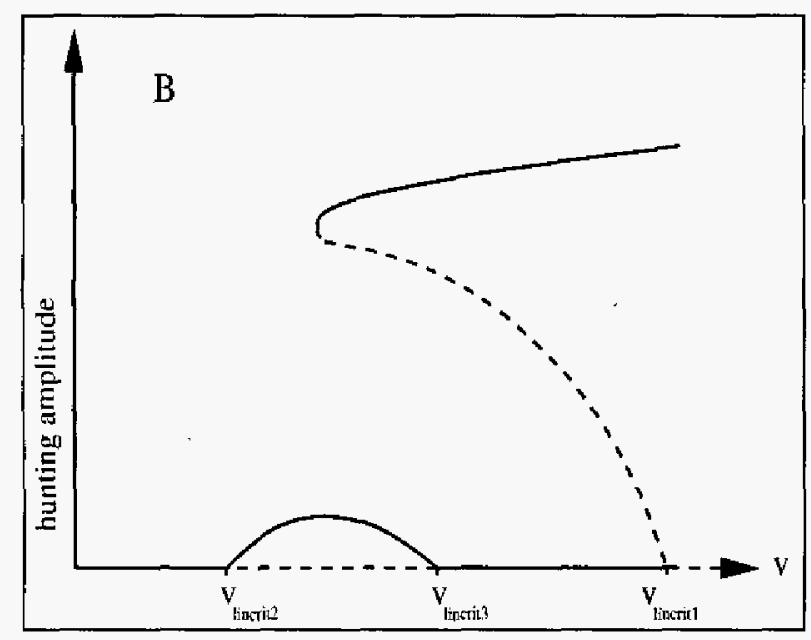

Figure 8. SECTION B FROM FIG.6. SHOWING THE SUBCRITICAL HOPF BIFURCATION TOGETHER WITH THE SMALL AMPLITUDE STABLE PERIODIC SOLUTION

unstable, and a new asymptotically stable periodic solution with small amplitude has been created in the interval. For larger $R$ the amplitude of the new periodic solution and the values of the bifurcation points $v_{\text {incrit } 2}$ and $v_{\text {lincrit } 3}$ all grow until $v_{\text {lincrit } 1}$ and $v_{\text {lincrit3 }}$ coalesce and the new periodic solution 'hits' the unstable periodic solution that emerges from v'lincrit 1 . The details of what looks like a kind of 'exchange of stability' at this point is under

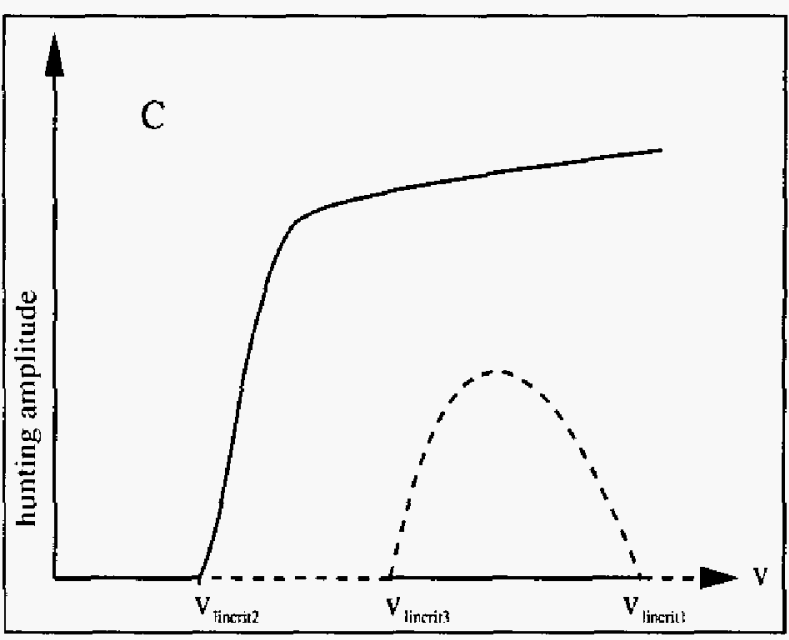

Figure 9. SECTION C FROM FIG.6 SHOWING THE SUBCRITICAL HOPF BIFURCATION TOGETHER WITH THE SMALL AMPLITUDE UNSTABLE PEAIODIC SOLUTION

investigation. For still larger values of $\mathrm{R}$ the saddle-node bifurcation point disappears, and the large amplitude hunting motion has become stable, and its bifurcation point is now a supercritical Hopf bifurcation. The small amplitude periodic solution has become unstable between the bifurcation points $v_{\text {lincrit } 3}$ and $v_{\text {lincrit } 1}$. while the stationary solution now has become asymptotically stable in the same speed interval. This situation is shown in Fig. 9 (section $\mathrm{C}$ ). For still higher values of $\mathrm{R}$ the amplitude of the small amplitude periodic solution decreases, while $v_{\text {lincrit } 3}$ and $v_{\text {lincrit } 1}$ approach each other, meet and finally annihilate each other. The existence of two periodic, stable solutions is demonstrated in the plots in Fig. 10.

The Hopf bifurcation points depend not only on the radius of the curve but also on the superelevation of the track. The critical speed for the Hopf bifurcation only changes little, but the Hopf bifurcation points tend towards lower radii of curvature, when the superelevation increases. The location of the Hopf bifurcation points depend on the contact surface geometry, and that in turn depends on the position of the wheelsets in the curve. In Table 1 we show the interval $\left[v_{\text {lincrit } 2}, v_{\text {lincrit }}\right]$ for selected values of the superelevation $\Phi$.

The onset of hunting in curves with growing speed depends on a delicate balance between the gravitational stiffness of the displaced wheelsets, the centrifugal and gravitational forces and the geometry of the entire wheel and rail profiles. In order to try to find some relations we have compared the yaw of the truck wheelsets versus the speed with the radial alignment for various radii. The superelevation $\Phi$ is again $2^{\circ}$. The results are shown in Fig. 11 and Fig. 12. It is interesting to notice that there might

Copyright (C) 2005 by ASME 


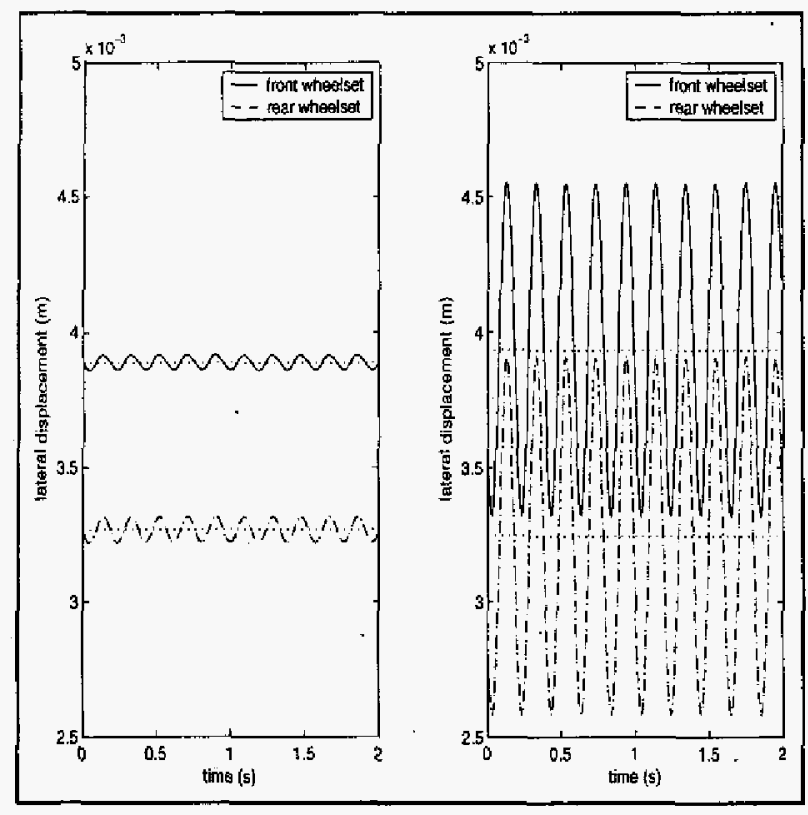

Figure 10. TWO PERIODIC STABLE SOLUTIONS AT $R=1230 \mathrm{~m}$ $V=50 \mathrm{~m} / \mathrm{s}$ AND $\Phi=2^{\circ}$

Table 1. THE INFLUENCE OF THE SUPEFELEVATION $\Phi$

\begin{tabular}{|cc|}
\hline$\Phi$ & $R_{\text {crit }}$ \\
\hline $0^{o}$ & {$[1265,1267.5]$} \\
$2^{o}$ & {$[1226.5,1226.6]$} \\
$3^{o}$ & {$[1207.5,1210]$} \\
$4^{o}$ & {$[1112.5,1115]$} \\
$6^{o}$ & {$[997.5,1000]$} \\
\hline
\end{tabular}

be a connection between the start of the periodic motion and the speed at which the position of a wheelset becomes radial. For low speeds both the leading and the trailing wheelsets have a larger yaw angle than the radial, meaning that they tend to steer the wheelsets out of the curve away from the center. The tendency is most pronounced for the trailing wheelset. In spite of the larger initial yaw angle the trailing axle will align radially first and then tend to steer the axle towards the center of the curve, when the speed increases. The leading axle follows suit. When the radius is larger than $1235 \mathrm{~m}$ hunting starts before any of the axles reaches a radial position.

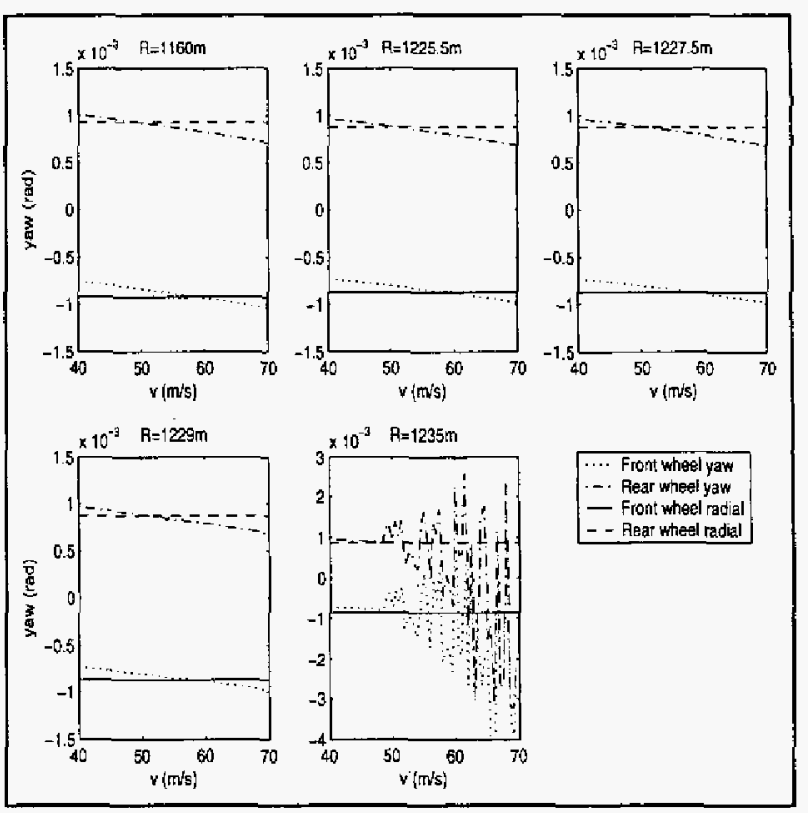

Figure 11. THE YAW ANGLES OF THE LEADING AND TRAILING AXLES VERSUS THE SPEED. $\Phi=2^{\circ}$

\section{Conclusion}

In 1983 Neil Cooperrider visited The Technical University of Denmark and had the opportunity to ride in the cab on one of the Danish 'Lyntog' - a fast $(140 \mathrm{~km} / \mathrm{h})$ diesel trainset, which basically was the same as the famous German TEE VT 11 trains. After the ride he reported to one of the authors (HT) that he had noticed a hunting motion in a couple of curves, through which the train had travelled with its maximum speed. On the rest of the line the train was running very calmly. It was noteworthy that the track condition was excellent all the way, and the hunting took place in curves with large radii - larger than $1000 \mathrm{~m}$. Neil Cooperrider expected that to happen, but he had never experienced it before, and he offered no explanation.

True and Birkedal Nielsen [5] found that the critical speed in curves is lower than on straight track, but their results were limited to curve radii smaller than $600 \mathrm{~m}$. In these curves the critical speeds were higher than the maximum speed allowed by the UIC limits on the maximum uncompensated lateral acceleration in curves.

Petersen and Hoffmann [6] calculated the critical speeds in curves with radii larger than $600 \mathrm{~m}$. They found that in curves with large radii the critical speed may be lower than the maximum allowed speed and also lower than the critical speed on straight track, so the vehicle may hunt in the curves only. That explains Neil Cooperrider's observation. 


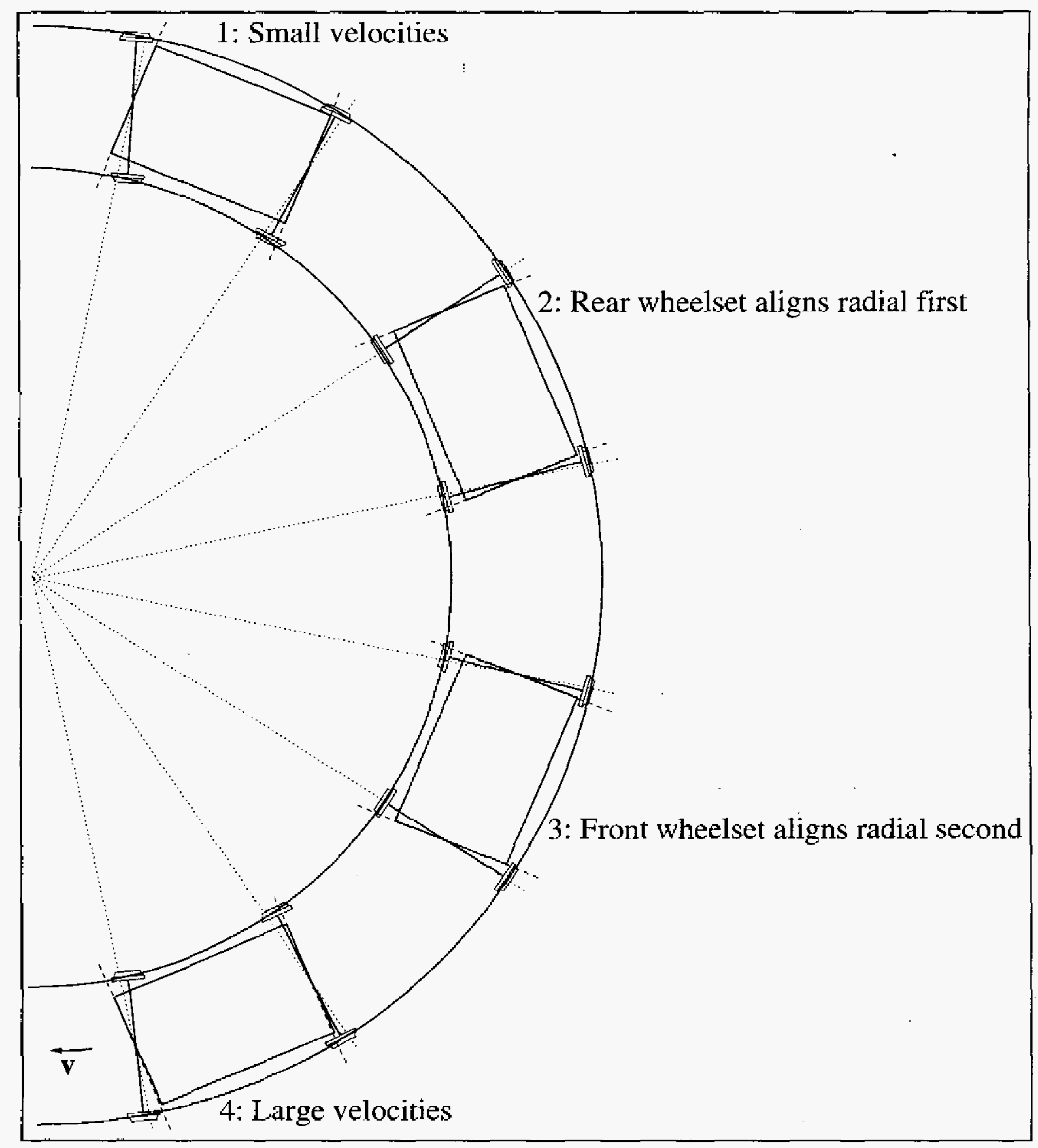

Figure 12. SCHEMATIC PLOT OF THE POSITION OF THE WHEELSETS AND THE TRUCK FRAME RELATIVE TO THE RADIAL LINE AT DIFFERENT SPEEDS. THE SPEED INCREASES FROM POSITION 1 TO POSITION 4 
This result has implications for the design parameters of railway vehicles, since it means that the reduction of the critical speed in curves must be taken into consideration in the design of the vehicle.

Petersen and Hoffmann [6] also found the jump of the linear critical speed - the Hopf bifurcation - down to the nonlinear critical speed that lakes place at a certain combination of the radius of the curve and the superelevation in the curve. In the present contribution we investigated the dynamics of the curving Coopertider truck in an interval around the radius, where the jump occurs, and hoped to relate the jump to a sudden change in the position of the truck on the track. The jump is connected with an interesting development of a new small amplitude periodic motion for speeds smaller than the original Hopf bifurcation. The creation of the new periodic motion seems to be connected with the speeds at which first the trailing and then the leading wheelset in the truck crosses the radial line. At larger curve radii the new periodic motion 'moves up' towards the original Hopf bifurcation. At a certain radius the two periodic motions - the unstable large amplitude motion and the stable small amplitude motion meet in what supposedly is - a kind of transcritical bifurcation. The bifurcation takes place in the bifurcation set with respect to changes of the radius. For still larger radii the former subcritical Hopf bifurcation turns supercritical, and the periodic branch becomes asymptotically stable. The small amplitude periodic motion now exists supercritically and is unstable. In tum the stationary solution has gained asymptotic stability in the speed interval where it coexists with both the periodic solutions.

We found no discontinuities in the position of the wheelsets as a function of the speed and the radius of the curve. Therefore the jump from the subcritical to the supercritical Hopf bifurcation is related to the smooth process of a kind of transcritical bifurcation in the bifurcation set.

We intend to continue our studies in this area. The details of what looks like a kind of transeritical bifurcation is of theoretical interest. It concerns the neighborhood of the radius where the two different periodic solutions meet. We would also like to extend our results, which were limited to radii beween $600 \mathrm{~m}$ and $2000 \mathrm{~m}$, to smaller radii and to radii tending towards infinity. It is necessary in order to establish the connection between our results and the results found by True and Birkedal Nielsen [5] for the smaller radii and verify the postulate on page 4 . We intend to extend our investigation to the case of maximum superelevation. Since the guiding forces are large in the curves, it is important to calculate the wheel-rail contact forces in the curves for as well the stationary as for the periodic motions. The results must be related to the position of the truck in the curves. The wheel/rail contact forces are, however, influenced by the dynamics of the track, so the 'rigid track assumption' in this work must then be dropped. The contact force problem will be investigated in a recent cooperation between the authors and the Polish Academy of Sciences.
The influence of the wheelbase and the stiffness of the primary suspension on the dynamics needs to be investigated. Highspeed trains have trucks with larger wheelbases than the $2.1 \mathrm{~m}$ of the Cooperrider truck, and the results of this work apply mainly to trains running fast through curves with large radii. The wheelbase and the stiffness of the primary suspension are supposedly the two most important parameters in this case, but it is of course desirable to examine the quasi-stationary curving dynamics of a wider range of existing truck constructions.

\section{REFERENCES}

[1] Kalker, J. J., 1991. "Wheel-rail rolling contact theory". In Mechanics and Fatigue in Wheel/Rail Contact, Proceedings of the Third International Conference on Contact Mechanics and Wear of Rail/Wheel Systems, S. Grassie, Ed., Elsevier, pp. 243-261.

[2] Klingel, W., 1883. "Über den Lauf der Eisenbahnwagen auf gerader Bahn". Organ für die Fortschritte des Eisenbahnwesens in technischer Beziehung, Neue Folge, 20 (4) , pp. 113123.

[3] Huilgol, R., 1978. "Hopf-Friedrichs bifurcation and the hunting of a railway axle". Quarterly of Applied Mathematics, 36, pp. 85-94.

[4] Knothe, K., and Böhm, F., 1999. "History of stability of railway and road vehicles". Vehicle System Dynamics, 31 (5-6) , pp. 283-323.

[5] True, H., and Birkedal Nielsen, J., 1999. "On the dynamics of steady curving of railway vehicles". In Proceedings 6th Miniconf. on Vehicle System Dynamics, Identification and Anomalies, I. Zobory, Ed., Technical University of Budapest, Technical University of Budapest, pp. 73-81.

[6] Petersen, D., and Hoffmann, H., 2002. Curving Dynamics of Railway Vehicles. Student report, IMM, The Technical University of Denmark, DK-2800 Kgs.Lyngby, Denmark. May.

[7] Cooperrider, N., 1972. "The hunting behavior of conventional railway trucks". ASME Journal of Engineering and Industry, 94, pp. 752-762.

[8] Shen, Z., Hedrick, J., and Elkins, J,, 1984. "A comparison of alternative creep-force models for rail vehicle dynamical analysis". In Proceedings 8th IAVSD Symposium on Vehicle System Dynamics, Swets \& Zeitlinger, Lisse, pp. 59 J-605.

[9] True, H.. 1999. "On the theory of nonlinear dynamies and its applications in vehicle systems dynamics". Vehicle System Dynamics, 31 (5-6) , pp. 393-421.

\section{ACKNOWLEDGMENT}

The support of this work by Railnet, Denmark and Banverket, Sweden is gratefully acknowledged. 